\title{
Expression of the Jimpy Gene in the Spinal Cords of Heterozygous Female Mice. I. An Early Myelin Deficit Followed by Compensation
}

\author{
William P. Bartlett and Robert P. Skoff \\ Department of Anatomy, Wayne State University School of Medicine, Detroit, Michigan 48201
}

The jimpy mutation results in severe hypomyelination throughout the CNS of hemizygous male mice. In the female carrier of the jimpy gene, partial hypomyelination is predicted as a consequence of genetic mosaicism resulting from random $X$-chromosome inactivation. The spinal cord of the female carrier was studied morphologically to determine (1) if hypomyelination is present, (2) the manner in which a possible myelin deficit is expressed, and (3) the extent, if any, of compensation.

The spinal cords of 14- to 15-d-old heterozygotes were found to be hypomyelinated. A deficit of $31 \%$ in the amount of myelin as compared to controls was detected in these young carriers by point-counting stereology. By the end of the first month the deficit was $12 \%$, and after the fifth month complete recovery had occurred. These results demonstrate that the neuroglial cells are capable of compensating totally for the jimpy defect over a several month period.

The reduction in the amount of myelin at 2 weeks postnatally is due to the ensheathment of fewer axons than normal and the formation of myelin sheaths that are thinner than normal. It is not due to a significant reduction in amount of axoplasm and a corresponding decrease in amount of myelin. This finding indicates that overall brain development is not retarded but that expression of the jimpy gene selectively affects the glial cells. Our morphologic studies also suggest that the neuron is not the target of the jimpy mutation. One line of evidence for this statement is that virtually all the axons are partially ensheathed, a condition that should not occur if $50 \%$ of the neurons are defective in the mosaic.

The coexistence of both normal and defective cells within the same cell population and the apparent sparing of the neuron makes the female carrier of the jimpy gene an excellent model for studying mechanisms of compensation and plasticity of neuroglial cells.

Jimpy (jp) is a sex-linked recessive gene that produces severe hypomyelination throughout the CNS of hemizygous male mice (Sidman et al., 1964). The affected jimpy males begin to exhibit body tremors by postnatal day 10 and usually die before day 30. The cause of the myelin deficit is not known although there have been numerous biochemical (e.g., Barbarese et al., 1979; Campagnoni et al., 1972; Carnow et al., 1984; Hogan, 1977; Meier et al., 1974) and morphological (e.g., Farkas-Bargeton et al., 1972; Meier and Bischoff, 1975; Privat et al., 1982; Skoff, 1976, 1982; Wolf et al., 1981) studies.

Due to the lethal nature of the jimpy gene in the males, the mutation is maintained in the heterozygous female. The female

Received June 3, 1985; revised Mar. 10, 1986; accepted Apr. 1, 1986.

This work was supported by NIH Research Grants NS 15338, NS 18883, and NS 18898 .

Correspondence should be addressed to William P. Bartlett, Ph.D., Department of Anatomy, Wayne State University School of Medicine, 540 East Canfield Avenue, Detroit, MI 48201.

Copyright (C) 1986 Society for Neuroscience $0270-6474 / 86 / 102802-11 \$ 02.00 / 0$ carrier is a mosaic and should express the jimpy gene according to Lyon's hypothesis of X-chromosome inactivation (Lyon, 1961, 1972). Expression of the jimpy gene in the female carrier was first described in the optic nerve by Skoff and Nowicki-Montgomery (1981). In the optic nerve of the young carrier, patches of unmyelinated axons are adjacent to areas of myelinated fibers. These patches of unmyelinated fibers may encompass a large portion of the cross-sectional area of the optic nerve and persist into adulthood (R. P. Skoff, unpublished observations).

In contrast to the nerve, patches of unmyelinated axons are not obvious in the brain and spinal cord (Rosenfeld and Friedrich, 1984; Skoff, 1982). In biochemical studies, however, measurements of myelin components are reduced $30-60 \%$ in the young female carrier (Benjamins et al., 1984; Kerner and Carson, 1984). Morphologically, a reduced amount of myelin has been demonstrated in the anterior commissure of the young carrier (Rosenfeld and Fricdrich, 1984). Howcver, it is unknown from these studies how the hypomyelination is expressed in the spinal cord and brain. Is the hypomyelination due to the ensheathment of fewer axons than normal, or to myelin sheaths that are thinner than normal? Another explanation for the deficit may be an overall retardation in brain development leading to a reduction in the amount of axoplasm in the carrier and a corresponding reduction in myelin.

The available evidence suggests that the deficit in the brain and spinal cord of the carrier is temporary and that the neuroglia are capable of compensating for the hypomyelination (Bartlett and Skoff, 1984; Benjamins et al., 1984; Rosenfeld and Friedrich, 1984; Skoff et al., 1985). The mechanisms utilized in compensation have not been explored.

We have undertaken the following study to answer 3 questions: (1) Is the spinal cord of the heterozygote hypomyelinated? (2) If so, in what manner is the deficit expressed? (3) Is the mosaic capable of compensation? The results from this study show that there is considerably less myelin in the spinal cords of 14-d-old carriers than controls. We provide evidence clearly showing that in the spinal cord the carrier is capable of recovering completely from the early deficiency in myelination.

\section{Materials and Methods}

Because of the jimpy gene's lethal nature, it is maintained in the female in the heterozygous state with Tabby $(\mathrm{Ta})$, a closely linked mutation modifying coat color (crossover frequency between $\mathrm{Ta}$ and jp is about $15 \%$; Wolf and Holden, 1969). Breeding pairs consisting of Tabby males $\left(\mathrm{Ta}^{+} / \mathrm{Y}\right)$ and female carriers of Tabby and jimpy (Tajp/++) were obtained from Jackson Laboratories (Bar Harbor, ME). Because of crossovers in the female parent, 4 different genotypes were obtained among the female offspring, parental $\mathrm{Tajp} / \mathrm{Ta}^{+}$and $\mathrm{Ta}^{+{ }^{++}}$; crossovers are $\mathrm{Ta}^{+} / \mathrm{Ta}^{+}$and ${ }^{+} \mathrm{jp} / \mathrm{Ta}^{+}$.

Mosaics were tentatively identified by the Tabby light brown fur coat and kink at the end of their tails ( Tajp/ $\mathrm{Ta}^{+}$) or the alternating bands of light and dark fur strips down their back $\left({ }^{+} \mathrm{jp} / \mathrm{Ta}^{+}\right)$. Definitive diagnosis for expression of the jimpy gene was made by the presence of patches of hypomyelinated axons in $1-\mu \mathrm{m}$-thick plastic sections of the optic nerve. Carriers of the jimpy gene can be identified at all ages, and patches 
of hypomyelination have been observed in 2-year-old carriers (R. P. Skoff, unpublished observations). Controls were female littermates $\left(\mathrm{Ta}^{+} /\right.$ $\left.{ }^{++}, \mathrm{Ta}^{+} / \mathrm{Ta}^{+}\right)$determined to be noncarriers only after extensive histological examination of both optic nerves failed to reveal evidence of mosaicism. In addition, several female offspring from noncarrier mothers were used as controls. A total of 25 mice were selected for analysis, 914 - to 15 -d-old mice (6 carriers, 3 normals), 1030 - to 34 -d-old mice (4 carriers, 6 normals), and 6120 - to 170 -d-old mice ( 3 carriers, 3 normals). Animals were anesthetized with chloral hydrate and sacrificed by intracardiac perfusion using $2 \%$ glutaraldehyde and $2 \%$ formaldehyde in $0.1 \mathrm{M}$ phosphate buffer. The entire optic nerve and middle cervical segments of the spinal cord were removed; the tissues were osmicated, dehydrated, and embedded in Araldite plastic using routine laboratory procedures.

The ventral column of the spinal cord was chosen for analysis because it contains a wide range of different sized fibers and a consistently superior fixation of myelin sheaths was obtained here in comparison to that in the dorsal column. Only the outer half of each ventral column, that portion adjacent to the rim of the ventral fissure, was used in this study (Fig. 1). This same area was reliably located from animal to animal and contained few dendrites that could be confused with unmyelinated axons.

Two different morphometric methods were used in this study. A point-counting stereologic method (Williams, 1977) was used to determine the percentage of total area occupied by myelin, the axoplasm of myelinated fibers, and the neuropil in light and electron micrographs. The area occupied by unmyelinated axoplasm was determined on electron micrographs only, which were of sufficient resolution to allow accurate identification. Three to 4 photomicrographs, enlarged to $\times 1800$ (encompassing 12,000 $\mu \mathrm{m}^{2}$ each), and 4-6 electron micrographs, enlarged to $\times 8500$ (encompassing $700 \mu \mathrm{m}^{2}$ each), were quantified from each spinal cord. The values derived from these micrographs were summed, and an average for the 4 different parameters measured was calculated for each animal using the Bioquant Point Counting Stereological Package.

In addition to point counting, digitization of individual axons and their myelin sheaths was performed on electron micrographs printed at approximately $\times 15,000$. From the spinal cords of the 2-week-old and 1-month-old mice, 4-6 micrographs were randomly selected from the same sampling area used for point-counting stereology (Fig. 1). All unmyelinated and myelinated axons were digitized in each micrograph. For each fiber, the perimeter of the axolemma was digitized along with the perimeter of the inner and outer lamellae of the compacted portion of the myelin sheath. The area of compact myelin was calculated by subtracting the area enclosed by the inner lamellae of myelin from that of the outer lamellae of myelin. Student's $t$ test was used to determine statistical significance.

\section{Results}

\section{Myelin deficit and recovery}

To determine the existence of hypomyelination in the carrier, the percentage area occupied by myelin in light and electron micrographs of the ventral column was determined by pointcounting stereology. In the 14- to 15 -d-old carriers, measurement of light micrographs employing stereology revealed that myelin sheaths occupied $24 \%$ of the area analyzed in the carriers compared to $35 \%$ in controls. The difference in percentages represents a $31 \%$ deficit in the myelin content of the mosaic relative to controls (Fig. 2). Point-counting analysis of electron micrographs showed that myelin sheaths occupied $20 \%$ in the carrier compared to $29 \%$ in controls, a reduction of $31 \%$ in mosaics $(p<0.005)$. The magnitude of the myelin deficit revealed by both methods was remarkably similar, given the fact that light and electron micrographs were used. A close correlation between the data derived from light and electron micrographs was also found for the other parameters (Fig. 2). Since the 2 methods produced similar results, we will discuss only the data obtained from the electron micrographs.

The myelin deficit in the 14- to 15-d-old carrier was most apparent along the periphery of the ventral columns adjacent to the pia (Fig. 3, $A$ and $B$ ). Here, numerous small areas or

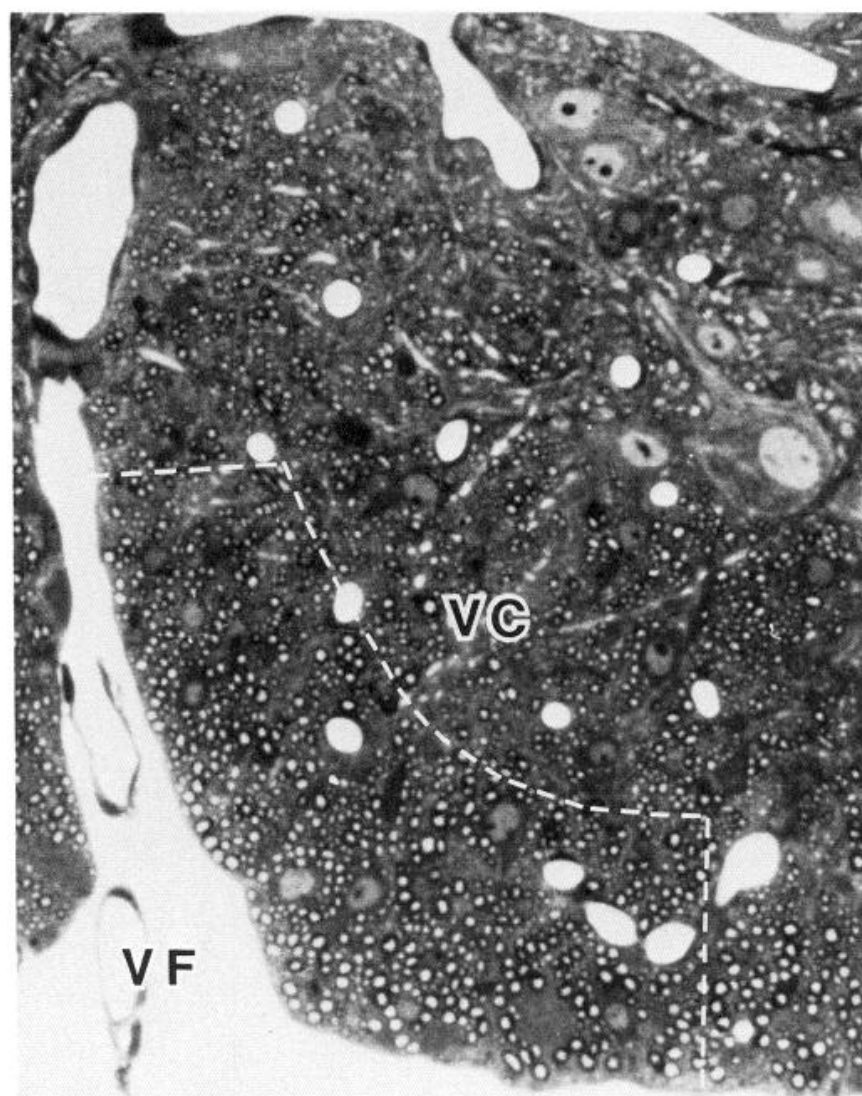

Figure 1. Photomicrograph of the ventral column $(V C)$ adjacent to the ventral fissure $(V F)$ in a spinal cord from a 14-d-old normal mouse Dotted line outlines the sampling area from which all light and electron micrographs were taken for morphometric analysis.

"patches" of hypomyelinated fibers were obvious. In electron micrographs, these subpial areas were found to contain many unmyelinated and thinly myelinated axons (Fig. 4). The majority of axons in the carrier had considerably thinner myelin sheaths than comparably sized axons in controls (Fig. 5).

Hypomyelination, while still detectable in 30- to 34-d-old carriers, was not as great as that found at younger ages. In the ventral column of the carrier, myelin sheaths occupied $37 \%$ of the area analyzed, compared to $41 \%$ in controls (Fig. 2). This represented a $10 \%(p<0.05)$ reduction in the amount of myelin in older carriers compared to the $32 \%$ deficit in the 14 - to 15 d-old carriers. Spinal cords from these older animals lacked the unmyelinated patches seen in the 14- to 15-d-old carriers (Fig. 6).

In mice 120-170 days old, the difference in the amount of myelin in spinal cords of carriers and controls was less than $2 \%$ (Fig. 2). Within 4-5 months the carrier had completely compensated for the myelin deficit.

\section{Axoplasm}

The deficit in the amount of myelin in the 14- to 15-d-old carrier could have been due to a reduction in the amount of axoplasm and a corresponding loss in myelin. However, point-counting stereology of electron micrographs reveals that total axoplasm, that of unmyelinated and myelinated axons combined, occupied $31 \%$ of the area analyzed in the carrier compared to $35 \%$ in controls (Fig. 2), a nonsignificant difference of less than $12 \%$ $(p>0.1)$. This suggests that a reduction in the amount of axoplasm was not the cause of the extensive hypomyelination found in the 14- to 15-d-old carrier. 

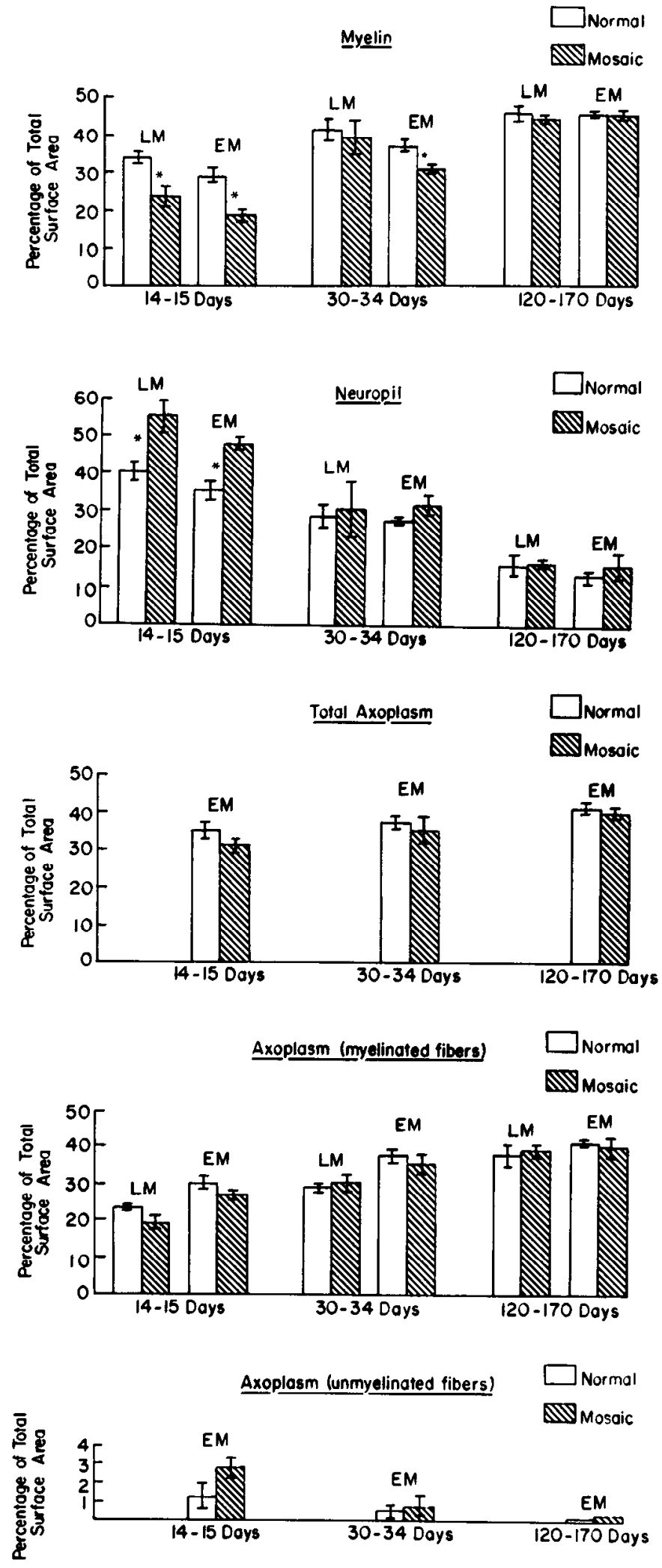

Figure 2. Percentage of total area occupied by myelin sheaths, neuropil, and axoplasm in light $(L M)$ and electron $(E M)$ micrographs of spinal cords from carriers and control mice as determined by pointcounting stereology. Myelin occupies 33\% ( $p<0.005$, asterisk) less area in the 14- to 15-d-old carrier than in controls. This deficit is reduced to $10 \%(p<0.05$, star) in 30 - to $34-\mathrm{d}$-old carriers and is completely compensated for by 120-170 d. No statistically significant difference was found in the total amount of axoplasm in carriers as compared to
Although the total amount of axoplasm was similar to that found in controls, the distribution of myelin between myelinated and unmyelinated axons was altered. This was shown by the large increase in the area occupied by unmyelinated axoplasm and in the decrease in area occupied by myelinated axoplasm (Fig. 2). Unmyelinated axoplasm occupied $2.8 \%$ of the area analyzed in the carrier compared to $1.2 \%$ in controls $(p<0.01)$. In the carrier, myelinated axoplasm occupied $29 \%$ of the area analyzed compared to $31 \%$ in controls, a difference of less than $7 \%(p>0.1)$. Thus, while there was no significant decrease in the total amount of axoplasm in the 14- to 15-d-old carrier, more axoplasm was found devoid of myelin sheaths than in controls.

The total amount of axoplasm in 30 - to 34 - and 120- to 170 d-old carriers was not significantly different than that found in age-matched controls (Fig. 2). Additionally, the area occupied by unmyelinated and myelinated axoplasm in these older carriers was essentially the same as that found in controls. The relatively large axons devoid of myelin found in the younger carriers (Fig. 4) were not present in these older carriers. This observation is a further indication that compensation for the myelin deficit occurred.

\section{Neuropil}

A significant increase in the area occupied by glial cell bodies and their processes was present in the 14- to 15-d-old heterozygotes. The neuropil occupied $48 \%$ of the area analyzed in the carrier compared to $37 \%$ in controls, an increase of $30 \%$ in the mosaic relative to control (Fig. 2). This hypertrophy of the neuropil was greatest in the outer portion of the ventral column adjacent to the pia (Figs. $3 B$ and 4), corresponding to the strips of hypomyelinated fibers found at this age in the carrier. In some micrographs from this portion of the ventral column the glial cell bodies and their processes were found to occupy up to $56 \%$ more area than that present in controls. The relative contribution of oligodendrocytes and astrocytes to the hypertrophy was not determined.

The hypertrophy of the neuropil in the young carrier could have been an artifact due to a reduction in the overall size of the ventral column accompanying the myelin deficit. If the mosaic cord was reduced in size, the neuropil would occupy a greater percentage of the total area than controls, but it would not have undergone any actual enlargement. However, measurements of the thickness of the ventral columns from controls and carriers revealed no significant difference between them.

The hypertrophy of the neuropil was reduced considerably in older carriers. In 30- to 34-d-old carriers the neuropil occupied $12 \%$ more area than controls and in 120- to 170 -d-old carriers it was only $10 \%$ above control levels (Fig. 2). The greatest reduction in this hypertrophy occurred between days 15 and 3034 , overlapping the period of most rapid compensation of the myelin deficit.

\section{Digitization of individual myelinated fibers}

Electron micrographs of the carrier spinal cord showed that myelin sheaths appeared thinner than those of comparably sized axons in controls (compare Fig. $3 A$ with $B$, and Fig. 4 with Fig. $5)$. To determine the severity of this reduction in the size of myelin sheaths in the carrier, the area occupied by an individual axon and its surrounding myelin sheath was digitized.

Electron micrographs of the ventral column adjacent to the pial surface from 14- to 15-d-old control and carrier mice were

controls at any time. The neuropil is $30 \%$ larger in young carriers than controls; however, by $120-170 \mathrm{~d}$ this hypertrophy has been reduced considerably. 

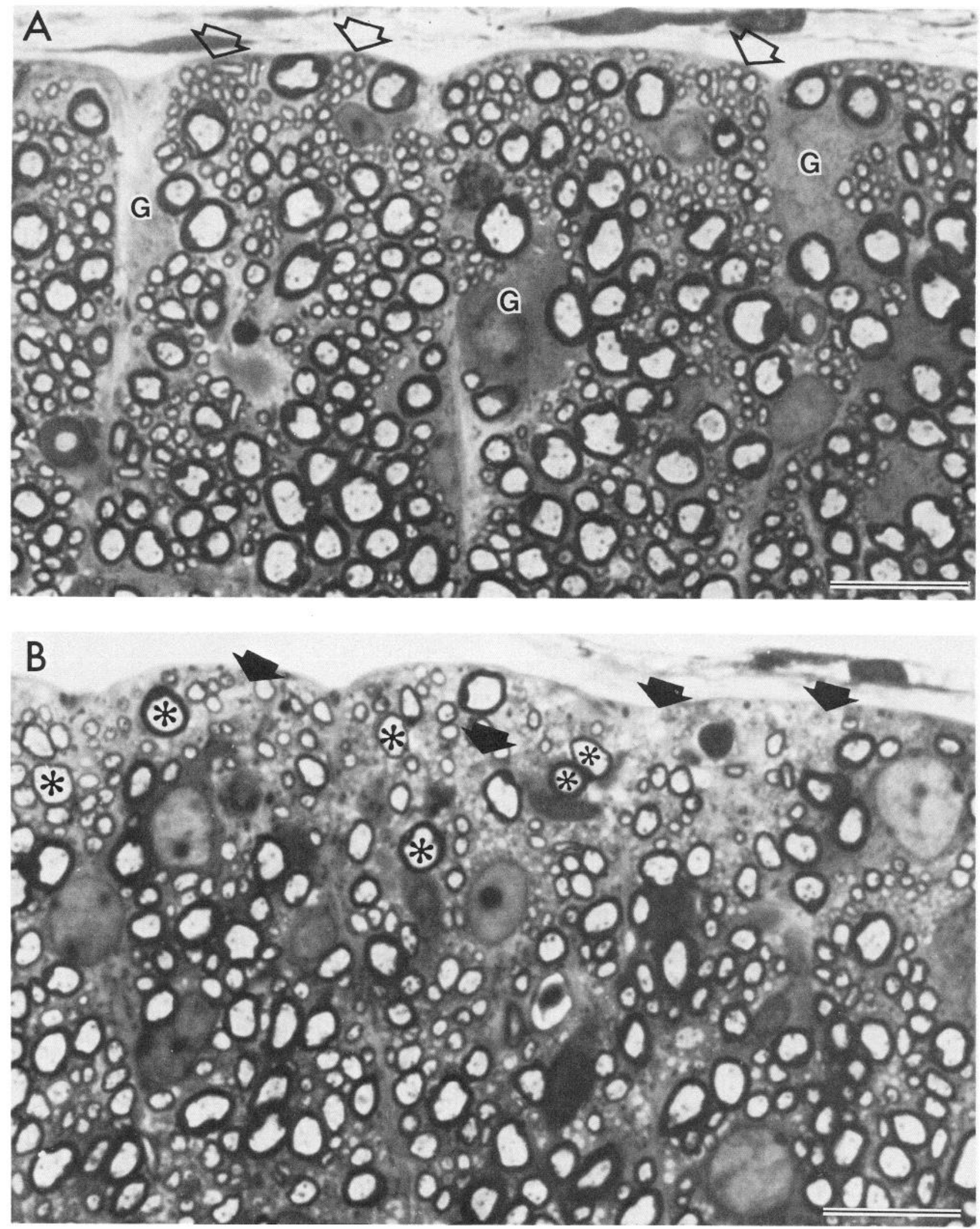

Figure 3. Photomicrographs of the ventral column adjacent to the ventral median fissure in the spinal cord of a normal $(A)$ and carrier $(B) 14$ to 15 -d-old mice. $A$, In control spinal cords, myelinated fibers are present just beneath the glia limitans (open arrowheads). Those areas free of myelin are occupied by glial perikarya and their processes $(G)$. B. In 14- to 15-d-old carrier spinal cords, a strip of white matter with little or no myelin is often evident along the glia limitans (arrowheads). Axons of similar size often have myelin sheaths of varying thickness (asterisks). Bars, $25 \mu \mathrm{m}$. 


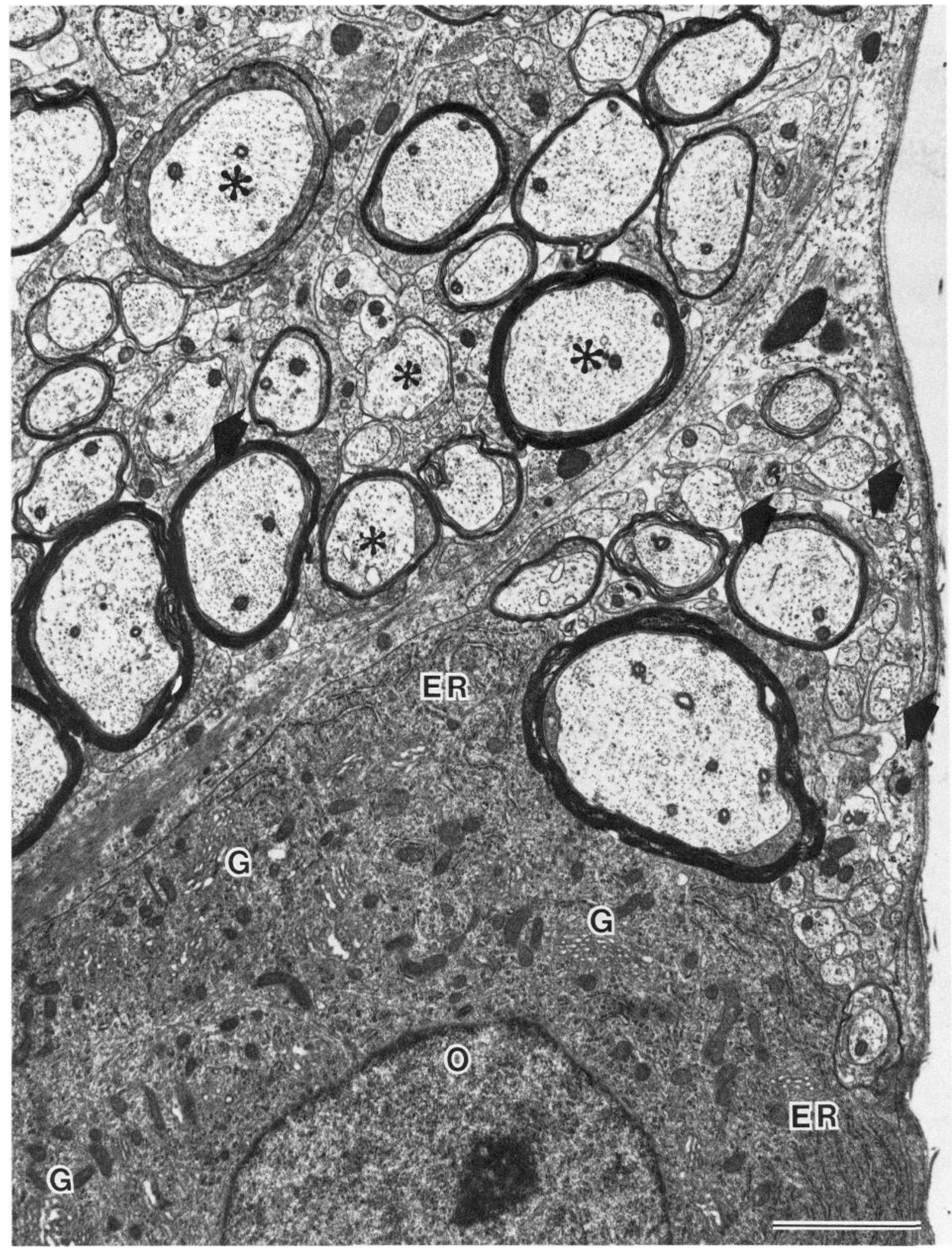

Figure 4. Electron micrograph of the spinal cord from a 14-d-old carrier. Unensheathed axons (arrowheads) are more numerous and also larger than those in controls (see Fig. 5). Those axons with myelin often have smaller sheaths than comparably sized control axons. In addition, the thickness of the myelin sheath varies considerably from axon to axon and does not always appear related to axonal diameter (asterisks). The profile of a large oligodendrocyte $(O)$ shows a moderately dense cytoplasm with numerous cisternae of the Golgi apparatus $(G)$ and of rough endoplasmic reticulum $(E R)$. Bar, $2 \mu \mathrm{m}$. 


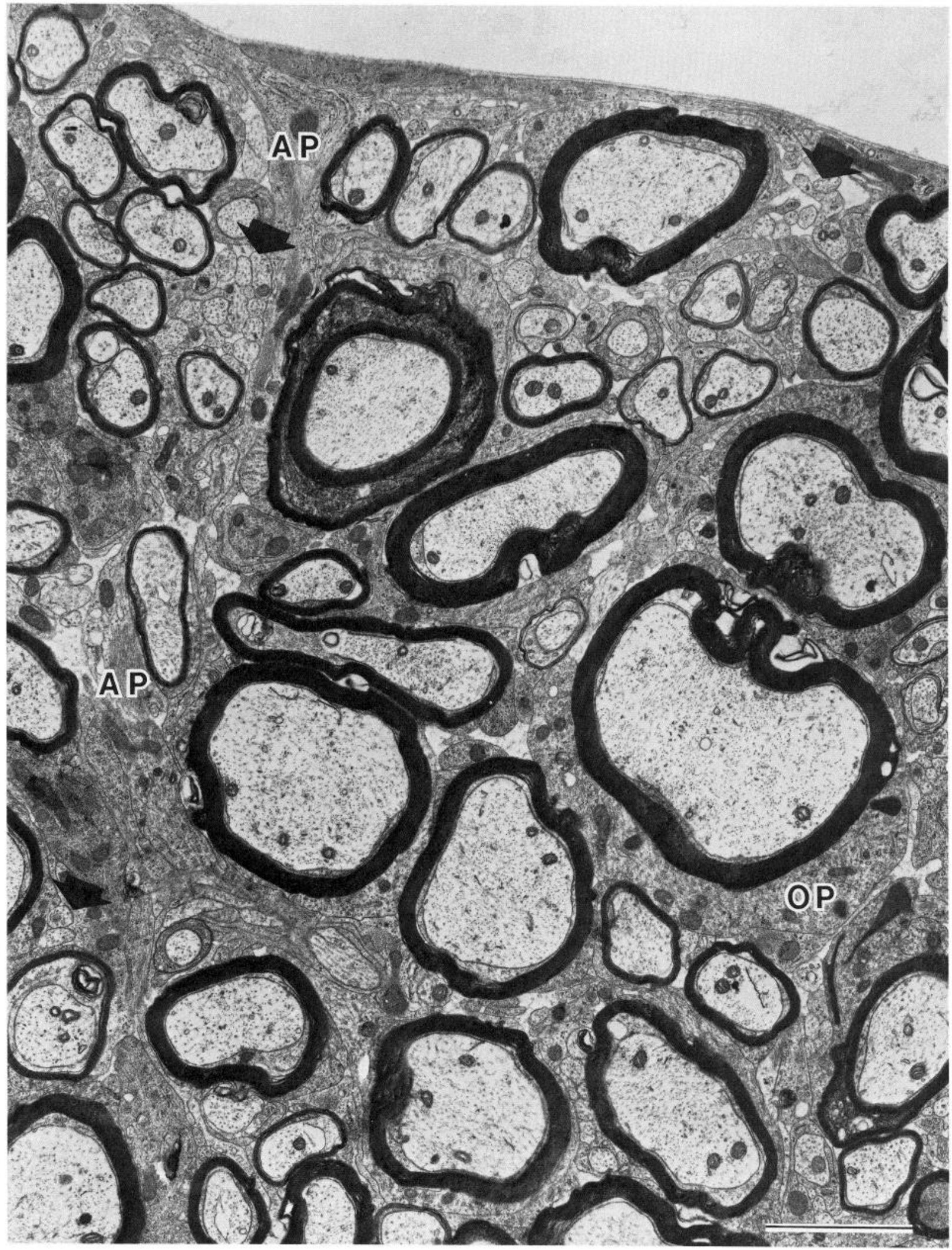

Figure 5. Electron micrograph of the spinal cord from a 14-d-old normal mouse. Only the smallest neurites are unmyelinated (arrowheads), and axons of similar size have myelin sheaths of comparable thickness. The neuropil is composed of astrocytic $(A P)$ and oligodendrocytic processes, some of which are actively myelinating axons $(O P)$. Bar, $2 \mu \mathrm{m}$. 

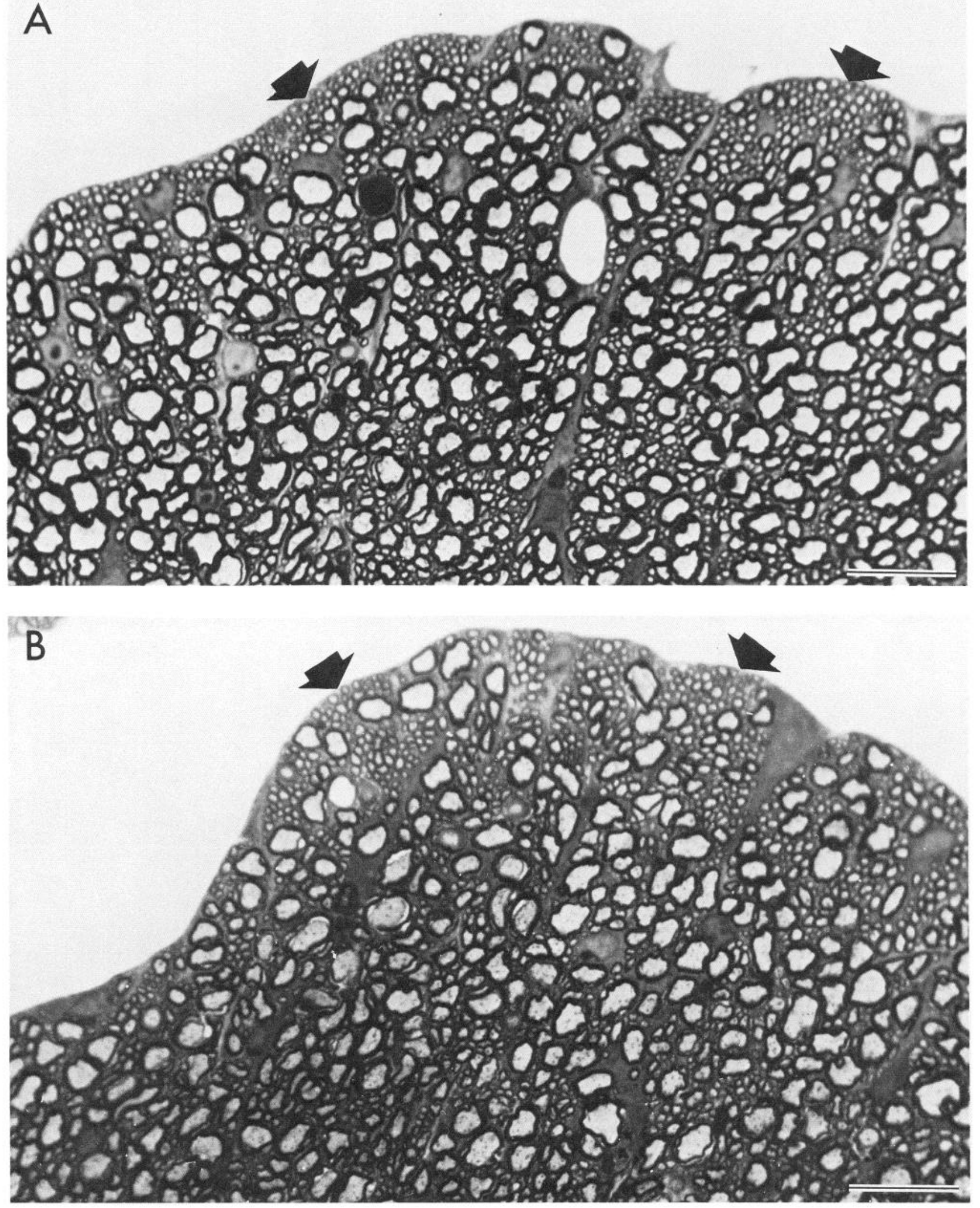

Figure 6. Photomicrographs of the ventral columns adjacent to the ventral median fissure in normal $(A)$ and carrier $(B)$ spinal cords of 30 -d-old mice. Areas of hypomyelinated axons are absent at this age in carrier spinal cords $(B$, arrowheads). In addition, the distribution and appearance of the myelinated fibers are similar in the carrier and in controls $(A)$. Bar, $20 \mu \mathrm{m}$. 
Table 1. Comparison of the average sizes of axons and myelin sheaths of myelinated fibers digitized from carrier and control mice

\begin{tabular}{|c|c|c|c|c|c|c|c|c|}
\hline & \multicolumn{4}{|c|}{ 14- to 15-d-old mice } & \multicolumn{4}{|c|}{ 30- to 34-d-old mice } \\
\hline & $\begin{array}{l}\text { No. of } \\
\text { fibers }\end{array}$ & $\begin{array}{l}\text { Area of } \\
\text { myelin }\end{array}$ & $\begin{array}{l}\text { Area of } \\
\text { axoplasm }\end{array}$ & $\begin{array}{l}\text { Ratio of } \\
\text { myelin/ } \\
\text { axoplasm }\end{array}$ & $\begin{array}{l}\text { No. of } \\
\text { fibers }\end{array}$ & $\begin{array}{l}\text { Area of } \\
\text { myelin }\end{array}$ & $\begin{array}{l}\text { Area of } \\
\text { axoplasm }\end{array}$ & $\begin{array}{l}\text { Ratio of } \\
\text { myelin/ } \\
\text { axoplasm }\end{array}$ \\
\hline Controls & 456 & 1.4 & 1.7 & 0.82 & 902 & 2.0 & 2.2 & 0.91 \\
\hline \multirow[t]{2}{*}{ Carriers } & 887 & $1.0^{a}$ & 1.8 & 0.55 & 484 & 1.9 & 2.4 & 0.79 \\
\hline & $\begin{array}{l}\% \text { diffe } \\
\text { axop }\end{array}$ & $\begin{array}{l}\text { the ratic } \\
\text { arrier/col }\end{array}$ & pyelin to & $33 \%$ & \multicolumn{3}{|c|}{$\begin{array}{l}\% \text { difference in the ratio of myelin to } \\
\text { axoplasm (carrier/control) }\end{array}$} & $13 \%$ \\
\hline
\end{tabular}

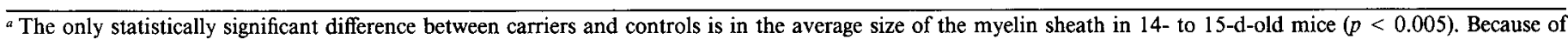
the wide range in the size of fibers analyzed, the SDs are larger than the average values and are not shown.

selected randomly for analysis, and the areas of all the axons and their myelin sheaths were digitized on each micrograph (see Materials and Methods). The area of axons in the carrier was similar in size to those of controls (Table 1), but their myelin sheaths were $29 \%$ smaller on average. When the ratio of the amount of myelin to axoplasm was determined for carriers and controls, it was reduced $33 \%$ in the heterozygote.

The deficit did not affect all fibers equally (Fig. $7 \mathrm{~A}$ ). In general, the larger the axon, the greater the myelin deficit. Axons larger than $2 \mu \mathrm{m}^{2}$ (approximately $0.9 \mu \mathrm{m}$ in diameter) had an average of $39 \%$ less myelin than comparably sized controls. In contrast, the myelin sheaths of axons less than $2 \mu \mathrm{m}^{2}$ in area were only $8 \%(p>0.2)$ smaller in the carriers.

In 30- to 34-d-old carriers, the average sizes of axons and myelin sheaths were not significantly different than those of controls (Table 1). In these older carriers the deficit in the myelin to axoplasm ratio was only $13 \%$ compared to the $33 \%$ reduction in the younger carriers. Furthermore, only the very largest of fibers, those axons $8-32 \mu \mathrm{m}^{2}$ in area, had a deficit in myelin sheath size greater than $10 \%$ (Fig. $7 \mathrm{~B}$ ). These results clearly show that by the end of the first postnatal month, the carrier had compensated almost completely for the myelin deficit.

The myelin sheaths surrounding axons from 120- to 170-dold mice could not be digitized accurately because of poor fixation. However, there appeared to be no obvious differences in the size of myelin sheaths between controls and carriers at this age.

\section{Discussion}

\section{$X$-chromosome inactivation mosaicism}

In female eutherian mammals, only one X-chromosome in somatic lines remains activated after early embryonic stages. This $\mathrm{X}$-chromosome inactivation is a regulatory mechanism that eliminates the effects of aneuploidy in somatic tissue and allows for normal germinal function. Mosaicism often occurs because of random inactivation of an X-chromosome during early embryonic development (Lyon, 1961, 1972).

The advantage of mosaics in the study of biological problems is that normal and mutant cells coexist within the same cell population. For this reason, mosaics have been used to analyze many different developmental processes, including patterns of migration, cell lineages, and cell differentiation (e.g., Nesbitt and Gartler, 1971). The female carrier of the jimpy gene is a mosaic, and she can be exploited to analyze a number of developmental processes that are not permitted with the hemizygous males. As an example, the normal life span of the heterozygote jimpy permits extended studies to determine the amount and mechanisms of compensation employed by the functional cells. This project was undertaken to determine if the cord of young mosaics is hypomyelinated, its manner of expression, and whether compensation occurs in the older animals.

\section{Expression of the myelin deficit}

The exact percentage of cells that express the jimpy mutation may vary between individual mosaics due to the random nature of X-chromosome inactivation. Lyon's hypothesis $(1961,1972)$ predicts that in a reasonably large population of cells, this value will be approximately $50 \%$. Experimental data suggest heterozygotes usually contain more or less equal numbers of the 2 cell populations in a particular tissue (McLaren, 1976). Thus, the myelin deficit should be approximately $50 \%$ before compen-

A

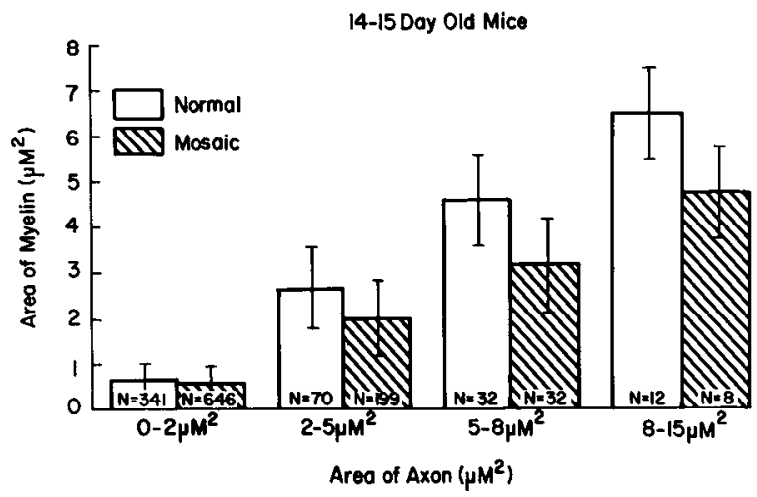

B

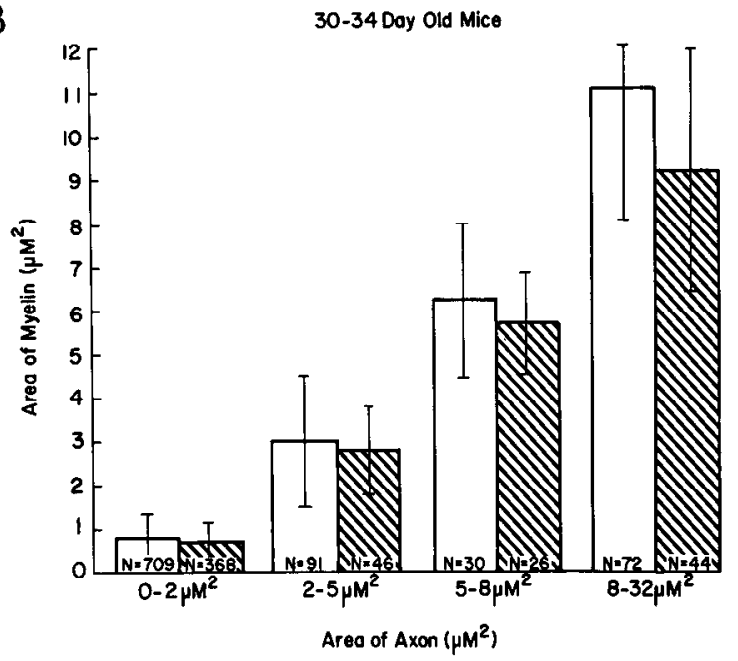

Figure 7. Relationship between the size of myelin sheaths and axons in myelinated fibers from 14- to 15-d-old $(A)$ and 30- to 34-d-old $(B)$ carrier and control spinal cords. In the 14- to 15 -d-old carrier $(A)$, axons greater than $2 \mu \mathrm{m}^{2}$ have significantly $(p<0.005)$ less myelin than controls. However, even the largest axons in 30 - to 34 -d-old carriers $(B)$, those greater than $8 \mu \mathrm{m}^{2}$, do not have significantly smaller $(p>0.1)$ myelin sheaths than comparably sized control axons. 
sation begins. However, in the 2-week-old mosaics we found a $31 \%$ reduction in the myelin content of spinal cords as compared to controls. In addition, the variation in myelin content between mosaics is small and similar to that of controls. The lack of significant variation and the smaller than expected deficit among the 6 mosaics at 2 weeks suggests that some compensation has already occurred in the spinal cords of these animals. A myelin deficit closer to $50 \%$ and more individual variation might be detected if the myelin content of younger mosaics were determined. However, unambiguous identification of the mosaics, which is based on the presence of patches of hypomyelinated axons in the optic nerve, is difficult in animals much younger than 2 weeks of age.

The amount of myelin in the 1-month-old mosaics was only $10 \%$ less than controls, and by the end of the fifth month, the myelin deficit was not detectable. These findings indicate that carriers of the jimpy gene are compensating completely for the myelin deficit. An alternate explanation for our results may be that only those mosaics that have inherited a very large percentage of normal cells are capable of compensation and that by chance these were the mosaics selected for analysis. This is an unlikely possibility since in this study we demonstrate that nearly complete compensation has occurred in the spinal cords of 7 out of 7 older mosaics and that some compensation has probably already occurred in the 62 -week-old mosaics analyzed. In addition, the variation in myelin content between mosaics is no different than age-matched controls. This suggests that the mosaic can compensate for variation that may occur in the initial ratio of normal to defective cells in the very young mosaic spinal cord and that this variation is not a critical determinant of the degree of recovery that eventually occurs in the adult.

Our demonstration of hypomyelination in the spinal cord of the young mosaic is in agreement with recent biochemical (Benjamins et al., 1984; Kerner and Carson, 1984; Skoff et al., 1985) and morphologic studies (Bartlett and Skoff, 1984; Rosenfeld and Friedrich, 1984) of female heterozygotes. Benjamins and coworkers have shown that in the brains of 14-d-old carriers the activity of enzymes associated with myelination, ceramide galactosyl transferase (CGT) and cerebroside sulfotransferase, and the levels of myelin basic protein (MBP) are reduced to 40$80 \%$ that of normal female littermates. The activity of CGT is similarly depressed in the spinal cord of young mosaics (Skoff et al., 1985). Kerner and Carson (1984) have found the levels of MBP, proteolipid protein, and $2^{\prime}, 3^{\prime}$-cyclic nucleotide- $3^{\prime}-$ phosphohydrolase in the hemispheres of young mosaics reduced $60-70 \%$ that of controls. In a morphometric study of the anterior commissure of the female heterozygote, Rosenfeld and Friedrich (1984) found a $33 \%$ deficit in myelin at $45 \mathrm{~d}$.

All of these studies have demonstrated hypomyelination in the young mosaics, but there is disagreement as to whether older mosaics are capable of recovery. Benjamins and her coworkers have shown that the activity of CGT increases rapidly both in brain and spinal cord and reaches near normal levels by the end of the first month (Benjamins et al., 1984; Skoff et al., 1985). Rosenfeld and Friedrich (1984) found no difference in the amount of myelin in the anterior commissure in mosaic and controls by $145 \mathrm{~d}$. In contrast to these findings, Kerner and Carson (1984) found no evidence of compensation occurring in cerebral hemispheres of 60-d-old carriers. The reason for this difference in results is not readily apparent.

The hypomyelination in the mosaic spinal cord could be due to a number of factors, including an overall retardation in brain development. In malnutrition (Wiggins, 1982) and lead intoxication (Tennekoon et al., 1979) there is a generalized delay in the increase of axon caliber and a corresponding decrease in myelin sheath thickness. If this were the case in the mosaic, the ratio of axoplasm to myelin would be essentially normal. This situation is obviously not occurring since the average size of axons in the mosaics is not significantly different from normals, while the ratio of axoplasm to myelin sheath area is sharply reduced. This finding shows that myelin sheaths in the mosaic are inappropriately small for the axon. An important conclusion can be drawn from this information: The axons apparently develop normally but myelinogenesis is preferentially retarded. What, then, is the basis of the myelin deficiency?

Our findings indicate that fewer axons in the mosaic are being ensheathed than in controls and that many of these sheaths are abnormally thin. Numerous, unmyelinated axons of sufficiently large caliber to be ensheathed are observed ultrastructurally in the 2 -week-old mosaics. This situation is also reflected quantitatively in the increased amount of unmyelinated axoplasm present in the younger mosaics compared to controls. As for the possibility that the sheaths are thinner than normal, myclinated axons in the mosaic have sheaths, that are, on average, $29 \%$ smaller than those of control axons. This reduction is not distributed equally between the different sized fibers. In general, the larger the axon, the greater the reduction. Fibers with axons greater than $2 \mu \mathrm{m}^{2}$ have $39 \%$ less myelin surrounding them than fibers in control cords. Axons smaller than $2 \mu \mathrm{m}^{2}$ have only an $8 \%$ deficit. Our interpretation for this finding is that ensheathment of these largest fibers was delayed in early development, when compensation is just beginning. The smaller fibers are less severely affected because their ensheathment begins later in development when compensation is well underway.

\section{Mechanisms of compensation}

The jimpy gene is on the maternal $\mathrm{X}$-chromosome $\left(\mathrm{X}^{\mathrm{m}}\right)$; thus, in cells in the mosaic that express the jimpy defect, it is the paternal $\mathrm{X}$-chromosome $\left(\mathrm{X}^{\mathrm{p}}\right)$ that has been inactivated. It is possible that the compensation found in the mosaic is due to the reactivation of the paternal or normal $X$-chromosome $\left(\mathrm{X}^{\mathrm{p}}\right)$ in the defective cells. This is an unlikely situation in mammalian cells. In mammalian cells, the inactivated X-chromosome appears to be very stable (Gartler and Riggs, 1983). Histochemical studies on clones from heterozygotes with enzymatic deficiencies in the levels of hypoxanthine phosphoribosyltransferase (Kahan and DeMars, 1975) or glucose 6-phosphate dehydrogenase (Migeon, 1972) show that reactivation is a rare event. Thus it is unlikely that reactivation of the paternal X-chromosome $\left(\mathrm{X}^{\mathrm{p}}\right)$ in jimpy cells contributes significantly to compensation in the mosaic.

It is more likely that compensation in the mosaic reflects the plasticity of the normal oligodendrocytes, those expressing the paternal $\mathrm{X}$-chromosome $\left(\mathrm{X}^{\mathrm{p}}\right)$, in responding to states of abnormal hypomyelination. Between the second and fourth postnatal weeks, the amount of myelin produced in the mosaic is increased considerably above control levels. This increased rate can be demonstrated by comparing the increase in size of axons to the increase in the size of their myelin sheaths during this period in mosaics to controls. The average size of axons increases about the same, $33 \%$ for the mosaic and $29 \%$ for controls, over this 2 week interval. However, the size of the myelin sheath increases $90 \%$ in the heterozygotes in comparison to $43 \%$ for the controls. This increase indicates that the oligodendrocytic population in the mosaics has generated twice as much myelin as those in the controls during the same period. Before considering how the oligodendrocytes accomplish this task, it is worth reflecting upon the constraints imposed upon the oligodendrocyte population if this cell is the target of the mutation. If oligodendroglia are the primary target, theoretically only $50 \%$ of this population should be functional at early stages of development. Yet they are capable of correcting completely for the defect over time. A limited number of compensatory mechanisms are available to the oligodendrocyte. These include the formation of additional cells of their own type, making internodal segments of myelin twice as long as they normally do, or myelinating more axons 
per oligodendrocyte than normal. Some or all of these mechanisms may be at work in the mosaic. In association with these mechanisms, an increased rate of production of myelin components by individual oligodendrocytes may be involved. The presence of numerous oligodendroglia with extremely large perikarya filled with organelles in the mosaic cord suggests that the capacity of an individual oligodendrocyte to produce myelin components may actually be increased in an abnormal situation. Our biochemical findings that CGT is increased above control levels in the $30 \mathrm{~d}$ mosaic cord support this interpretation (Skoff et al., 1985).

One of the possible mechanisms of compensation that has been extensively studied is the capacity of oligodendrocytes to divide in response to demyelinating lesions. In models of experimentally induced demyelination/remyelination, oligodendrocytes have been shown to arise from undifferentiated precursors (Ludwin, 1979) and mature oligodendroglia (Arenella and Herndon, 1984; Ludwin, 1984). The proliferative response of oligodendrocytes to hypomyelination in the mosaic is presently under study in our laboratory. Preliminary studies from our laboratory utilizing ${ }^{3} \mathrm{H}$-thymidine autoradiography indicates that there is indeed a significant increase in the number of dividing oligodendroglia in the young mosaic spinal cord compared to controls (W. P. Bartlett and R. P. Skoff, unpublished observations).

\section{Neuroglial hypertrophy}

The reduction in the volume of myelin in the mosaics is accompanied by an increase in the volume of neuropil. At 2 weeks of age, glial perikarya and their processes occupy $30 \%$ more volume than in controls, $12 \%$ in the 1 -month-old mice, and $10 \%$ by 5 months of age. The contribution made by astrocytes and oligodendrocytes to the glial hypertrophy in the spinal cords was not determined in the present study, but both types are probably represented. An astroglial hypertrophy in the optic nerves of young mosaics has been demonstrated with GFAP immunocytochemistry (Hatfield and Skoff, 1982). The gliosis in the optic nerves of the mosaic is often similar in appearance and intensity to that seen in affected males (Hatfield and Skoff, 1982). These gliosed patches were not observed in the brain or cord, but GFAP immunoreactivity more intense than in female controls was clearly apparent in certain tracts such as the internal capsule. The number of astrocytes immunostained was increased, as was the number of astroglial processes. While our evidence is circumstantial, it suggests that an astroglial hypertrophy is present in many of the mosaic fiber tracts during development and that it correlates with the degree of the hypomyelination. If the reduction in the amount of neuropil in the spinal cord of the older mosaics is in part attributable to a reduction in astroglial processes, then this would suggest that an astroglial hypertrophy is a reversible process in certain circumstances. This is an intriguing concept because it suggests that a dynamic relationship exists between myelinogenesis and astrocytic gliosis.

\section{Neuron as the target of the jimpy mutation}

The mosaic should be considered not only a model for the study of glial plasticity, but it can also provide important clues about which cell type is the primary target of the jimpy gene. One possibility is that the neuron is the site of the mutation, and the axons are unable to signal the oligodendrocyte to establish contact and/or maintain a myelin sheath. If the neuron were at fault, one would predict, according to the hypothesis of Lyon (1972), that approximately half of the neurons should be normal and become myelinated along their entire length and the other half of the axons in the mosaic remain unmyelinated. This is clearly not the case with the mosaic since the vast majority of the axons become ensheathed. Furthermore, individual axons in spinal cord and optic nerve may have both myelinated and unmyelinated segments. Where individual axons in the mosaic cord have been sectioned longitudinally and examined in the $\mathrm{EM}$, they may be bare for relatively long distances, i.e., $9 \mu \mathrm{m}^{2}$ or more (Bartlett and Skoff, 1984, and unpublished observations). It is clear from these observations that 2 distinct populations of axons, one myelinated and the other unmyelinated, do not exist in the mosaics. This condition makes it highly unlikely that the neuron is the site of the mutation.

\section{Jimpy mosaic as a model to study glial plasticity}

The apparent sparing of the neuron in the jimpy mosaic and the restriction of the defect to the neuroglia provide a distinct advantage over other experimental systems in which myelination has been delayed or demyelination produced. In those paradigms in which myelination is retarded, such as lead intoxication (Tennekoon et al., 1979; Toews et al., 1980) and malnutrition (Wiggins, 1982), axons are also reduced in size. In these systems, maturation of the whole brain is apparently delayed. In the case of the demyelinating/remyelinating models, such as cuprizone (Blakemore, 1973), ethidium bromide (Yajima and Suzuki, 1979), and triethyl tin (Blaker et al., 1981), the axoplasm often initially shows tremendous distortion and swelling, suggesting a dual effect upon both glia and neurons. These multiple effects make it difficult to separate primary from secondary effects in these systems. It is also important to note that recovery in these models is rarely complete (Ludwin, 1981; Ludwin and Maitland, 1984), whereas it is in the mosaic. The mosaic, then, provides an excellent system to study the mechanisms of compensation utilized by neuroglia and to determine the extent of their plasticity during development. The information obtained from this system can then be compared to other systems.

\section{References}

Arenella, L. S., and R. M. Herndon (1984) Mature oligodendrocytes. Division following experimental demyelination in adult animals. Arch. Neurol. 41: 1162-1165.

Barbarese, E., J. H. Carson, and P. E. Braun (1979) Subcellular distribution and structural polymorphism of myelin basic protein in normal and jimpy mouse brain. J. Neurochem. 32: 1437-1466.

Bartlett, W. P., and R. P. Skoff (1984) Expression of mosaicism in spinal cords of jimpy heterozygous females. Soc. Neurosci. Abstr. 10: 426.

Benjamins, J. A., R. P. Skoff, and K. Beyer (1984) Biochemical expression of mosaicism in female mice heterozygous for the jimpy gene. J. Neurochem. 42: 487-492.

Blakemore, W. F. (1973) Demyelination of the superior cerebellar peduncle in the mouse induced by cuprizone. J. Neurol. Sci. 20:6372.

Blaker, W. D., M. P. Krigman, and P. Morell (1981) Effect of triethyl tin on myelination in the developing rat. J. Neurochem. 36:44-52.

Campagnoni, A. T., C. W. Campagnoni, A. L. Huang, and J. Sapugna (1972) Developmental changes in the basic protein of normal and jimpy mouse brain. Biochem. Biophys. Res. Commun. 46: 700-707.

Carnow, T. B., J. H. Carson, S. W. Brostoff, and E. L. Hogan (1984) Myelin basic protein gene expression in quaking, jimpy and myelin synthesis-deficient mice. Dev. Biol. 106: 38-44.

Farkas-Bargeton, E., O. Robain, and P. Mandel (1972) Abnormal glial maturation in the white matter in jimpy mice. An optical study. Acta Neuropathol. 21: 272-281.

Gartler, S. M., and A. D. Riggs (1983) Mammalian X-chromosome inactivation. Annu. Rev. Genet. 17: 155-190.

Hatfield, J. S., and R. P. Skoff (1982) GFAP immunoreactivity reveals astrogliosis in females heterozygous for jimpy. Brain Res. 250: 123131.

Hogan, E. L. (1977) Animal models of genetic disorders of myelin. In Myelin, P. Morrell, ed., pp. 489-520, Plenum, New York.

Kahan, B., and R. DeMars (1975) Localized depression on the human inactive X-chromosome in mouse-human cell hybrid. Proc. Natl. Acad. Sci. USA 72: 1510-1514. 
Kerner, A. L., and J. H. Carson (1984) Effect of jimpy mutation on expression of myelin proteins in heterozygous and hemizygous mouse brain. J. Neurochem. 43: 1706-1714.

Ludwin, S. K. (1979) An autoradiographic study of cellular proliferation in remyelination of the central nervous system. Am. J. Pathol. 95: 683-690.

Ludwin, S. K. (1981) Pathology of demyelination and remyelination. In Demyelinating Disease: Basic and Clinical Electrophysiology, S. G. Waxman and J. M. Ritchie, eds., pp. 123-168, Raven, New York.

Ludwin, S. K. (1984) Proliferation of mature oligodendrocytes after trauma to the central nervous system. Nature 308: 274-275.

Ludwin, S. K., and M. Maitland (1984) Long-term remyelination fails to reconstitute normal thickness of central myelin sheaths. J. Neurol. Sci. 64: 193-198.

Lyon, M. F. (1961) Gene action in the X-chromosome of the mouse (Mus musculus L.). Nature 190: 372-373.

Lyon, M. F. (1972) X-Chromosome inactivation and developmental patterns in mammals. Biol. Rev. 47: 1-35.

McLaren, A. (1976) Chimaeras versus mosaics. In Mammalian Chimaeras, Cambridge U. P., New York.

Meier, C., and A. Bischoff (1975) Oligodendroglial cell development in jimpy mice and controls: An electron microscopic study in the optic nerve. I. Neurol. Sci. 26: 517-528.

Meier, C., N. Herschkowitz, and A. Bischoff (1974) Morphological and biochemical observations in the jimpy spinal cord. Acta Neuropathol. 27: 349-362.

Migeon, B. R. (1972) Stability of X-chromosome inactivation in human somatic cells. Nature 239: 87-89.

Nesbitt, M., and S. M. Gartler (1971) The applications of genetic mosaicism to developmental problems. Annu. Rev. Genet. 5: 143162.

Privat, A., J. Valat, F. Lachapelle, N. Baumann, and J. Fulcrand (1982) Radioautographic evidence for the protracted proliferation of glial cells in the central nervous system of jimpy mice. Dev. Brain Res. 2: 411-416.

Rosenfeld, J., and V. L. Friedrich, Jr. (1984) Hypomyelination and recovery of the myelin deficit in heterozygous jimpy mice. Int. J. Dev. Neurosci. 2(1): 21-32.
Sidman, R. L., M. M. Dickie, and S. II. Appel (1964) Mutant mice (quaking and jimpy) with deficient myelination in the central nervous system. Science 144: 309-311.

Skoff, R. P. (1976) Myelin deficit in the jimpy mouse may be due to cellular abnormalities in astroglia. Nature 264: 560-562.

Skoff, R. P. (1982) Increased proliferation of oligodendrocytes in the hypomyelinated mouse mutant jimpy. Brain Res. 248: 19-31.

Skoff, R. P., and I. Nowicki-Montgomery (1981) Expression of mosaicism in females heterozygous for jimpy. Brain Res. 212: 175-181.

Skoff, R. P., D. S. Studzinski, and J. A. Benjamins (1985) Ceramide galactosyl transferase in brain and cord of mosaic jimpy mice. Am. Soc. Neurochem. Abstr. 16: 291.

Sturrock, R. R. (1980) Myelination of the mouse corpus callosum. Neuropathol. Appl. Neurobiol. 6: 415-420.

Tennekoon, G., C. S. Aitchison, J. Frangia, D. L. Price, and A. M. Goldberg (1979) Chronic lead intoxication: Effects on developing optic nerve. Ann. Neurol. 5: 558-564.

Toews, A. D., M. R. Krigman, D. J. Thomas, and P. Morrell (1980) Effect of inorganic lead exposure on myelination in the rat. Neurochem. Res. 5: 605-616.

Webster, H. deF., P. J. Reier, J. M. Matthieu, and R. H. Quarles (1976) Axonal abnormalities in optic nerves of jimpy mice. J. Neuropathol. Exp. Neurol. 35: 303.

Wiggins, R. C. (1982) Myelin development and nutritional insufficiency. Brain Res. Rev. 4: 151-175.

Williams, M. A. (1977) Quantitative Methods in Biology, ElsevierNorth Holland, Amsterdam.

Wolf, M. K., and A. B. Holden (1969) Tissue culture analysis of the inherited defect of central nervous system myclination in jimpy micc. J. Neuropathol. Exp. Neurol. 28: 195-213.

Wolf, M. K., G. B. Schwing, L. H. Adcock, and S. Billings-Gagliardi (1981) Hypomyelinated mutant mice. III. Increased myelination in mutant cerebellum co-cultured with normal optic nerve. Brain Res. 206: 193-197.

Yajima, K. and K. Suzuki (1979) Ultrastructural changes of oligodendroglia and myelin sheaths induced by ethidium bromide. Neuropathol. Appl. Neurobiol. 5: 49-62. 